\title{
The John-Nirenberg type inequality for non-doubling measures
}

\author{
by \\ Yoshiniro Sawano and Hitoshi Tanaka (Tokyo)
}

\begin{abstract}
X. Tolsa defined a space of BMO type for positive Radon measures satisfying some growth condition on $\mathbb{R}^{d}$. This new BMO space is very suitable for the CalderónZygmund theory with non-doubling measures. Especially, the John-Nirenberg type inequality can be recovered. In the present paper we introduce a localized and weighted version of this inequality and, as applications, we obtain some vector-valued inequalities and weighted inequalities for Morrey spaces.
\end{abstract}

1. Introduction. It is well known that the doubling property of the underlying measure is a basic condition in the classical Calderón-Zygmund theory of harmonic analysis. Recently, more attention has been paid to nondoubling measures. It has been shown that many results of this theory still hold without the doubling property.

Nazarov, Treil and Volberg developed the theory of singular integrals for certain non-doubling measures (growth measures), satisfying a growth condition given by (1.1) below, and investigated the analytic capacity on the complex plane $[3,4]$. Tolsa proved subadditivity and bi-Lipschitz invariance of the analytic capacity, which had been left open for a long time $[12,13]$. The research, stemming from their pioneer works using the modified maximal operator, has been developed in many ways. Tolsa defined for growth measures the RBMO (regular bounded mean oscillation) space, the Hardy space $H^{1}(\mu)$ and the Littlewood-Paley decomposition $[9,10]$. He also gave his $H^{1}(\mu)$ space in terms of the grand maximal operator [11]. He established a John-Nirenberg type inequality for his RBMO space. Deng, Han and Yang defined Besov spaces and Triebel-Lizorkin spaces and the present authors defined Morrey spaces and established some inequalities for growth measures $[1,2,7,8]$.

2000 Mathematics Subject Classification: Primary 42B35; Secondary 42B25.

Key words and phrases: non-doubling measure, John-Nirenberg inequality, RBMO, Morrey space, sharp maximal inequality. 
The purpose of the present paper is to introduce and prove a localized and weighted John-Nirenberg type inequality for growth measures. The result can be applied to obtain a vector-valued sharp maximal inequality and a weighted sharp maximal inequality for Morrey spaces.

We first recall some definitions and notations. By a "cube" $Q \subset \mathbb{R}^{d}$ we mean a compact cube whose edges are parallel to the coordinate axes. Its center will be denoted by $z_{Q}$ and its sidelength by $\ell(Q) . Q(x, l)$ denotes the cube centered at $x$ and of sidelength $l$. For $\varrho>0, \varrho Q$ denotes the cube concentric with $Q$ of sidelength $\varrho \ell(Q)$. Throughout this paper we assume that $\mu$ is a positive Radon measure satisfying the following growth condition:

$$
\mu(Q(x, l)) \leq C_{0} l^{n} \quad \text { for all } x \in \operatorname{supp}(\mu) \text { and } l>0,
$$

where $C_{0}$ and $n \in(0, d]$ are some fixed numbers. We emphasize that $\mu$ is not necessarily a doubling measure, that is, we do not assume the doubling inequality $\mu(2 Q) \leq C \mu(Q)$ for every cube $Q \subset \mathbb{R}^{d}$. We denote by $\mathcal{Q}(\mu)$ the set of all cubes $Q \subset \mathbb{R}^{d}$ with positive $\mu$-measure. We say that $Q \in \mathcal{Q}(\mu)$ is a doubling cube if $\mu(2 Q) \leq 2^{d+1} \mu(Q)$. Let $\mathcal{Q}(\mu, 2)$ denote the set of all doubling cubes. By the growth condition (1.1) there are a lot of big doubling cubes. More precisely, given any $Q \in \mathcal{Q}(\mu)$, we can find $j \in \mathbb{N}$ with $2^{j} Q \in \mathcal{Q}(\mu, 2)$. Given $Q \in \mathcal{Q}(\mu)$, we let $Q^{*}$ be the smallest doubling cube $R$ of the form $R=2^{j} Q$ with $j=0,1, \ldots$ Given two cubes $Q, R \in \mathcal{Q}(\mu)$ with $Q \subset R$, we set

$$
\delta(Q, R):=\int_{\ell(Q)}^{\ell\left(Q_{R}\right)} \frac{\mu\left(Q\left(z_{Q}, l\right)\right)}{l^{n}} \frac{d l}{l},
$$

where $Q_{R}$ denotes the smallest cube concentric with $Q$ and containing $R$.

The space of BMO type we use in the present paper is RBMO (regular bounded mean oscillation) introduced by Tolsa [9], which is a suitable substitute for the classical BMO space. A function $f \in L_{\text {loc }}^{1}(\mu)$ is said to be in RBMO if

$$
\begin{aligned}
\|f\|_{*}:= & \sup _{Q \in \mathcal{Q}(\mu)} \frac{1}{\mu\left(\frac{3}{2} Q\right)} \int_{Q}\left|f(x)-m_{Q^{*}}(f)\right| d \mu(x) \\
& +\sup _{\substack{Q \subset R \\
Q, R \in \mathcal{Q}(\mu, 2)}} \frac{\left|m_{Q}(f)-m_{R}(f)\right|}{1+\delta(Q, R)}<\infty,
\end{aligned}
$$

where $m_{Q}(f):=\mu(Q)^{-1} \int_{Q} f(x) d \mu(x), Q \in \mathcal{Q}(\mu)$. (Many other equivalent norms may be found in [9, Section 2].) An advantage of RBMO is that it satisfies the following John-Nirenberg inequality due to Tolsa.

Theorem 1.1 ([10, Section 3]). Let $f \in \operatorname{RBMO}$ and $Q \in \mathcal{Q}(\mu)$.

(i) There exist positive constants $C$ and $C^{\prime}$ independent of $f$ so that, for every $\lambda>0$ and every cube $Q$, 


$$
\mu\left\{x \in Q:\left|f(x)-m_{Q^{*}}(f)\right|>\lambda\right\} \leq C \mu\left(\frac{3}{2} Q\right) \exp \left(-\frac{C^{\prime} \lambda}{\|f\|_{*}}\right) .
$$

(ii) Let $q \in[1, \infty)$. Then there exists a constant $C$ independent of $f$ so that, for every cube $Q \in \mathcal{Q}(\mu)$,

$$
\left(\frac{1}{\mu\left(\frac{3}{2} Q\right)} \int_{Q}\left|f(x)-m_{Q^{*}}(f)\right|^{q} d \mu(x)\right)^{1 / q} \leq C\|f\|_{*} .
$$

The purpose of the present paper is to obtain a localized and weighted version of Theorem 1.1(ii) (Theorem 1.2 below). As an application, we extend Theorem 1.1(ii) to a vector-valued inequality (Corollary 3.3).

To describe our theorem, we need to introduce two maximal operators also due to Tolsa. For $f \in L_{\text {loc }}^{1}(\mu)$, the sharp maximal operator $M^{\sharp} f(x)$ is defined as

$$
\begin{aligned}
M^{\sharp} f(x):= & \sup _{x \in Q \in \mathcal{Q}(\mu)} \frac{1}{\mu\left(\frac{3}{2} Q\right)} \int_{Q}\left|f(x)-m_{Q^{*}}(f)\right| d \mu(x) \\
& +\sup _{\substack{x \in Q \subset R \\
Q, R \in \mathcal{Q}(\mu, 2)}} \frac{\left|m_{Q}(f)-m_{R}(f)\right|}{1+\delta(Q, R)},
\end{aligned}
$$

and $N f(x)$ is defined as

$$
N f(x):=\sup _{x \in Q \in \mathcal{Q}(\mu, 2)} m_{Q}(|f|) .
$$

It is well known that $N$ is a bounded operator on $L^{p}(\mu)$ with $p>1$. We denote by $\|N\|_{p}$ its operator norm. Since for every $Q \in \mathcal{Q}(\mu)$ the sequence $Q, 2^{-1} Q, 2^{-2} Q, \ldots$ contains infinitely many doubling cubes, we also have a pointwise estimate $|f(x)| \leq N f(x)$ for $\mu$-a.e. $x \in \mathbb{R}^{d}$. Denote by $N^{k}, k \in \mathbb{N}$, the $k$-fold composition of the operator $N$. Throughout the paper a weight $w$ will be a non-negative function on $\mathbb{R}^{d}$ satisfying the (mild) condition

$$
w \in L^{p_{0}}(\mu) \quad \text { for some } p_{0}>1 \text {. }
$$

For simplicity we denote $\int_{E} w(x) d \mu(x), E \subset \mathbb{R}^{d}$, by $w(E)$. For a weight function $w$ satisfying (1.2), we define

$$
W(x):=\sum_{k=1}^{\infty}(2 \beta)^{1-k} N^{k} w(x), \quad \beta \geq\|N\|_{p_{0}} .
$$

The following theorem is the main result of the present paper.

Theorem 1.2. Let $0<\alpha<1$ and $1 \leq q<\infty$. Suppose that a weight function $w$ satisfies (1.2). Then, for every $f \in L_{\mathrm{loc}}^{1}(\mu)$ and $Q_{0} \in \mathcal{Q}(\mu)$, there 
exists a constant $C$ independent of $f$ and $Q_{0}$ such that

$$
\left(\int_{Q_{0}}\left|f(x)-m_{\left(Q_{0}\right)^{*}}(f)\right|^{q} W(x)^{\alpha} d \mu(x)\right)^{1 / q} \leq C\left(\int_{\frac{3}{2} Q_{0}} M^{\sharp} f(x)^{q} W(x)^{\alpha} d \mu(x)\right)^{1 / q} .
$$

2. Proof of Theorem 1.2. The letter $C$ will be used for constants that may change from one occurrence to another. Constants with subscripts, such as $C_{0}$ and $C_{1}$, do not change in different occurrences.

Generations of cubes. Here we shall define generations of cubes and investigate their properties. It is a local version of [11] and our construction is based on a slightly modified form of the one given in [11].

LEMma 2.1. The following assertions hold.

(i) For $\varrho>1$ and $Q \in \mathcal{Q}(\mu)$, we have $\delta(Q, \varrho Q) \leq C_{0} \log \varrho$.

(ii) Let $Q \in \mathcal{Q}(\mu)$. Then $\delta\left(Q, Q^{*}\right) \leq C_{0} 2^{n+1} \log 2$.

(iii) Let $Q \in \mathcal{Q}(\mu), k_{0} \in \mathbb{N}$ and $\theta_{0}>0$. Suppose that, for some $\theta_{1}>0$,

$$
\theta_{0} \leq \mu(Q) \leq \mu\left(2^{k_{0}} Q\right) \leq \theta_{0} \theta_{1} .
$$

Then $\delta\left(Q, 2^{k_{0}} Q\right) \leq 2^{n} \log 2 \cdot \theta_{1} C_{0} c_{n}$, where $c_{n}:=\sum_{k=0}^{\infty} 2^{-n k}$.

Proof. Assertion (i) follows easily from the growth condition (1.1). We prove (ii). Let $Q^{*}=2^{k_{1}} Q$ with $k_{1} \in \mathbb{N} \cup\{0\}$. A dyadic argument shows that

$$
\delta\left(Q, 2^{k_{1}} Q\right)=\int_{\ell(Q)}^{\ell\left(2^{k_{1}} Q\right)} \frac{\mu\left(Q\left(z_{Q}, l\right)\right)}{l^{n}} \frac{d l}{l} \leq 2^{n} \log 2 \sum_{k=1}^{k_{1}} \frac{\mu\left(2^{k} Q\right)}{\ell\left(2^{k} Q\right)^{n}} .
$$

By the growth condition we have $d-n \geq 0$. The definitions of $Q^{*}$ and doubling cubes imply $2^{d+1} \mu\left(2^{k-1} Q\right) \leq \mu\left(2^{k} Q\right), k=1, \ldots, k_{1}$. These observations yield

$$
\delta\left(Q, 2^{k_{1}} Q\right) \leq 2^{n} \log 2 \cdot \frac{\mu\left(2^{k_{1}} Q\right)}{\ell\left(2^{k_{1}} Q\right)^{n}} \sum_{k=1}^{k_{1}}\left(2^{n-d-1}\right)^{k_{1}-k} \leq C_{0} 2^{n+1} \log 2 .
$$

It remains to prove (iii). It follows from a dyadic argument and assumption (2.1) that

$$
\begin{aligned}
\delta\left(Q, 2^{k_{0}} Q\right) \leq 2^{n} \log 2 \cdot \sum_{k=1}^{k_{0}} \frac{\mu\left(2^{k} Q\right)}{\ell\left(2^{k} Q\right)^{n}} & \leq 2^{n} \log 2 \cdot \theta_{1} \frac{\theta_{0}}{\ell(Q)^{n}} \sum_{k=1}^{k_{0}} 2^{-n k} \\
& \leq 2^{n} \log 2 \cdot \theta_{1} C_{0} c_{n},
\end{aligned}
$$

which concludes the proof. 
Given two cubes $Q \subset R$, we set

$$
\widetilde{\delta}(Q, R):=\int_{\ell(Q)}^{\ell\left(Q^{R}\right)} \frac{\mu\left(Q\left(z_{Q}, l\right)\right)}{l^{n}} \frac{d l}{l},
$$

where $Q^{R}$ denotes the largest cube concentric with $Q$ and contained in $R$. We will treat a point $x \in \operatorname{supp}(\mu)$ as if it were a cube with sidelength 0 . So, for $x \in \operatorname{supp}(\mu)$ and some cube $R \ni x$, the notations $\widetilde{\delta}(x, R)$ and $x^{R}$ make sense.

Let $C_{1}:=C_{0} 2^{n+1} \log 2$. Letting $Q_{0} \in \mathcal{Q}(\mu)$, we set $Q_{1}:=\frac{3}{2} Q_{0}$.

Lemma 2.2. Suppose that $A_{0}$ is large enough, say $A_{0}>3 C_{1}$. Then, for each $x \in Q_{0} \cap \operatorname{supp}(\mu)$ with $\widetilde{\delta}\left(x, Q_{1}\right)>A_{0}$, there exists some doubling cube $Q \subset Q_{1}$ centered at $x$ satisfying

$$
\left|\widetilde{\delta}\left(Q, Q_{1}\right)-A_{0}\right| \leq 2 C_{1}
$$

Proof. Let $R$ be a unique cube of the form $2^{-k} x^{Q_{1}}, k=1,2, \ldots$, such that

$$
\widetilde{\delta}\left(2 R, Q_{1}\right) \leq A_{0}<\widetilde{\delta}\left(R, Q_{1}\right)
$$

Then

$$
A_{0}<\widetilde{\delta}\left(R, Q_{1}\right)=\widetilde{\delta}(R, 2 R)+\widetilde{\delta}\left(2 R, Q_{1}\right) \leq C_{0} \log 2+\widetilde{\delta}\left(2 R, Q_{1}\right)
$$

This implies $2 C_{1} \leq \widetilde{\delta}\left(2 R, Q_{1}\right)$ and hence, by Lemma 2.1(ii), we conclude that $Q:=(2 R)^{*} \subset Q_{1}$. It follows from Lemma 2.1 (ii) again that $A_{0}<\widetilde{\delta}\left(R, Q_{1}\right)=$ $\widetilde{\delta}(R, Q)+\widetilde{\delta}\left(Q, Q_{1}\right) \leq 2 C_{1}+\widetilde{\delta}\left(Q, Q_{1}\right)$ and that $\widetilde{\delta}\left(Q, Q_{1}\right) \leq \widetilde{\delta}\left(2 R, Q_{1}\right) \leq A_{0}$ Thus, we obtain $\left|\widetilde{\delta}\left(Q, Q_{1}\right)-A_{0}\right| \leq 2 C_{1}$.

Let $m \in \mathbb{N}$ and $A>3 C_{1}$. Then we set

$$
\Omega_{m, A}:=\left\{x \in Q_{0} \cap \operatorname{supp}(\mu): \widetilde{\delta}\left(x, Q_{1}\right)>m A\right\}
$$

and

$$
\widehat{D}_{m, A}:=\left\{Q \in \mathcal{Q}(\mu, 2): Q \subset Q_{1}, z_{Q} \in \Omega_{m, A} \text { and }\left|\widetilde{\delta}\left(Q, Q_{1}\right)-m A\right| \leq 2 C_{1}\right\} \text {. }
$$

Lemma 2.3. Assume that $A$ is large enough. Let $P \in \widehat{D}_{m, A}$ and $P^{\prime} \in$ $\widehat{D}_{m+1, A}$.

(i) Suppose that $z_{P}=z_{P^{\prime}}$. Then $100 P^{\prime} \subset P$.

(ii) Suppose that $P \cap P^{\prime} \neq \emptyset$. Then $\ell\left(P^{\prime}\right) \leq \frac{1}{8} \ell(P)$.

Proof. (i) Suppose to the contrary that $P \subset 100 P^{\prime}$. Then since $\widetilde{\delta}\left(P^{\prime}, Q_{1}\right)$ $=\widetilde{\delta}\left(P^{\prime}, 100 P^{\prime}\right)+\widetilde{\delta}\left(100 P^{\prime}, Q_{1}\right)$ we obtain

$$
(m+1) A-2 C_{1} \leq \widetilde{\delta}\left(P^{\prime}, 100 P^{\prime}\right)+m A+2 C_{1} \leq C_{0} \log 100+m A+2 C_{1} .
$$

This implies $A \leq C_{0} \log 100+4 C_{1}$. Thus, we have $P \supset 100 P^{\prime}$ provided $A>C_{0} \log 100+4 C_{1}$. 
(ii) If $8 \ell\left(P^{\prime}\right)>\ell(P)$, then $P \subset 24 P^{\prime}$. As a result, defining $R:=$ $Q\left(z_{P}, 48 \ell\left(P^{\prime}\right)\right)$, we have $P, P^{\prime} \subset 24 P^{\prime} \subset R \subset 72 P^{\prime} \subset Q_{1}$, and hence

$$
\widetilde{\delta}\left(P^{\prime}, R\right) \leq \delta\left(P^{\prime}, 72 P^{\prime}\right) \leq C .
$$

We now claim that

$$
s:=\left|\widetilde{\delta}\left(P^{\prime R}, Q_{1}\right)-\widetilde{\delta}\left(R, Q_{1}\right)\right| \leq C .
$$

We decompose $s$ as

$$
\begin{aligned}
s= & \int_{\ell\left(P^{\prime R}\right)}^{\ell\left(P^{\prime Q_{1}}\right)} \frac{\mu\left(Q\left(z_{P^{\prime}}, l\right)\right)}{l^{n}} \frac{d l}{l}-\int_{\ell(R)}^{\ell\left(R^{Q_{1}}\right)} \frac{\mu\left(Q\left(z_{P}, l\right)\right)}{l^{n}} \frac{d l}{l} \mid \\
\leq & \int_{\ell\left(P^{\prime R}\right)}^{\ell(R)} \frac{\mu\left(Q\left(z_{P^{\prime}}, l\right)\right)}{l^{n}} \frac{d l}{l} \\
& +\left|\int_{\ell(R)}^{\min \left\{\ell\left(P^{\prime Q_{1}}\right), \ell\left(R^{Q_{1}}\right)\right\}}\left(\mu\left(Q\left(z_{P^{\prime}}, l\right)\right)-\mu\left(Q\left(z_{P}, l\right)\right)\right) \frac{d l}{l^{n+1}}\right| \\
& +\int_{\max \left\{\ell\left(P^{\prime} Q_{1}\right), \ell\left(R^{Q_{1}}\right)\right\}}\left(\frac{\mu\left(Q\left(z_{P^{\prime}}, l\right)\right)}{l^{n}}+\frac{\mu\left(Q\left(z_{P}, l\right)\right)}{l^{n}}\right) \frac{d l}{l} \\
= & : s_{1}+s_{2}+s_{3} .
\end{aligned}
$$

The integrals $s_{1}$ and $s_{3}$ are easily estimated above by some constant $C$. We bound $s_{2}$ from above by

$$
\int_{\ell(R)}^{\infty} \mu\left(Q\left(z_{P^{\prime}}, l\right) \triangle Q\left(z_{P}, l\right)\right) \frac{d l}{l^{n+1}}=\int_{\ell(R)}^{\infty}\left(\int_{\mathbb{R}^{d}} \chi_{Q\left(z_{P^{\prime}}, l\right) \triangle Q\left(z_{P}, l\right)}(x) d \mu(x)\right) \frac{d l}{l^{n+1}},
$$

where $\chi_{E}$ is the indicator function of a set $E \subset \mathbb{R}^{d}$. Let

$$
|x|_{\infty}:=\max \left\{\left|x_{1}\right|, \ldots,\left|x_{d}\right|\right\}, \quad x=\left(x_{1}, \ldots, x_{d}\right) \in \mathbb{R}^{d} .
$$

Then a simple geometric observation tells us that

$$
\begin{aligned}
& \chi_{Q\left(z_{P^{\prime}}, l\right) \triangle Q\left(z_{P}, l\right)}(x)=0 \\
& \quad \text { if } l \notin\left[\min \left\{\left|x-z_{P}\right|_{\infty},\left|x-z_{P^{\prime}}\right|_{\infty}\right\}, \max \left\{\left|x-z_{P}\right|_{\infty},\left|x-z_{P^{\prime}}\right|_{\infty}\right\}\right] .
\end{aligned}
$$

In view of this observation we obtain

$$
\begin{aligned}
s_{2} & \leq C \int_{\mathbb{R}^{d} \backslash P^{\prime}}\left|\frac{1}{\left|x-z_{P}\right|_{\infty}^{n}}-\frac{1}{\left|x-z_{P^{\prime}}\right|_{\infty}^{n}}\right| d \mu(x) \\
& \leq C \int_{\left|x-z_{P^{\prime}}\right|_{\infty} \geq \ell\left(P^{\prime}\right) / 2} \frac{\left|z_{P^{\prime}}-z_{P}\right|_{\infty}}{\left|x-z_{P^{\prime}}\right|_{\infty}^{n+1}} d \mu(x) .
\end{aligned}
$$


A routine dyadic argument gives

$$
\begin{aligned}
s_{2} & \leq C \sum_{k=0}^{\infty} \int_{2^{k-1} \ell\left(P^{\prime}\right) \leq\left|x-z_{P^{\prime}}\right|_{\infty} \leq 2^{k} \ell\left(P^{\prime}\right)} \frac{\left|z_{P^{\prime}}-z_{P}\right|_{\infty}}{\left|x-z_{P^{\prime}}\right|_{\infty}^{n+1}} d \mu(x) \\
& \leq C \frac{\left|z_{P^{\prime}}-z_{P}\right|_{\infty}}{\ell\left(P^{\prime}\right)} \leq C .
\end{aligned}
$$

This proves (2.3).

From the relation $\widetilde{\delta}\left(P^{\prime}, Q_{1}\right)=\widetilde{\delta}\left(P^{\prime}, R\right)+\widetilde{\delta}\left(P^{\prime R}, Q_{1}\right),(2.2)$ and (2.3) we have

$$
\begin{aligned}
\widetilde{\delta}\left(P^{\prime}, Q_{1}\right) & \leq \widetilde{\delta}\left(P^{\prime}, R\right)+\left|\widetilde{\delta}\left(P^{\prime R}, Q_{1}\right)-\widetilde{\delta}\left(R, Q_{1}\right)\right|+\widetilde{\delta}\left(R, Q_{1}\right) \\
& \leq \widetilde{\delta}\left(P, Q_{1}\right)+C_{2},
\end{aligned}
$$

where $C_{2}$ is some fixed big constant. Hence $(m+1) A \leq m A+4 C_{1}+C_{2}$. Thus, $\ell\left(P^{\prime}\right) \leq \frac{1}{8} \ell(P)$ provided $A>4 C_{1}+C_{2}$.

Let $m \in \mathbb{N}$ and choose $A$ large enough so that the conclusion of Lemma 2.3 holds. From Lemma 2.2 there exists a map $\Phi_{m}: \Omega_{m, A} \rightarrow \widehat{D}_{m, A}$ such that $z_{\Phi_{m}(x)}=x$ for all $x \in \Omega_{m, A}$. Fixing this collection $\left\{\Phi_{m}\right\}_{m \in \mathbb{N}}$ of maps, we set $Q_{x, m}:=\Phi_{m}(x)$. Then by the definition we have

$$
\left|\widetilde{\delta}\left(Q_{x, m}, Q_{1}\right)-m A\right| \leq 2 C_{1} .
$$

The cubes $Q_{x, m}, m \in \mathbb{N}, x \in \Omega_{m, A}$, are called cubes of the mth generation (relative to $Q_{0}$ ). The set of all cubes of the $m$ th generation will be denoted by $D_{m}$ and the set $\bigcup_{m} D_{m}$ will be denoted by $D$.

The weight $W$. Now we record some simple properties of the weight $W$ defined by (1.3). To begin with, we notice that $N$ is subadditive and $W$ satisfies the so-called $A_{1}$-condition:

$$
N W(x) \leq 2 \beta W(x) \quad \text { for } \mu \text {-a.e. } x \in \mathbb{R}^{d} .
$$

Indeed,

$$
\begin{aligned}
N W(x) & \leq \sum_{j=1}^{\infty}(2 \beta)^{1-j} N^{j+1} w(x)=2 \beta\left\{\sum_{j=1}^{\infty}(2 \beta)^{1-j} N^{j} w(x)-N w(x)\right\} \\
& \leq 2 \beta W(x) .
\end{aligned}
$$

Once we have established (2.4), the so-called $A_{\infty}$-property follows immediately.

Lemma 2.4. Let $\alpha \in(0,1)$ and $Q \in \mathcal{Q}(\mu, 2)$. Then

$$
\frac{W^{\alpha}(E)}{W^{\alpha}(Q)} \leq(2 \beta)^{\alpha}\left(\frac{\mu(E)}{\mu(Q)}\right)^{1-\alpha}
$$

for all $\mu$-measurable subsets $E \subset Q$. 
Proof. Using Hölder's inequality we have

$$
\begin{aligned}
W^{\alpha}(E) & =\int_{E} W(x)^{\alpha} d \mu(x) \leq\left(\int_{E} W(x) d \mu(x)\right)^{\alpha} \mu(E)^{1-\alpha} \\
& \leq \mu(Q)\left(\frac{W(Q)}{\mu(Q)}\right)^{\alpha}\left(\frac{\mu(E)}{\mu(Q)}\right)^{1-\alpha} .
\end{aligned}
$$

It follows from (2.4) that

$$
W^{\alpha}(E) \leq\left(\int_{Q} N W(x)^{\alpha} d \mu(x)\right) \cdot\left(\frac{\mu(E)}{\mu(Q)}\right)^{1-\alpha} \leq(2 \beta)^{\alpha} W^{\alpha}(Q) \cdot\left(\frac{\mu(E)}{\mu(Q)}\right)^{1-\alpha},
$$

which proves the lemma.

Proof of Theorem 1.2. Choose $A$ large enough so that the conclusion of Lemma 2.3 holds, and fix $D_{m}$ and $D$. Letting $F(x):=\left|f(x)-m_{\left(2 Q_{0}\right)^{*}}(f)\right|$, we consider the maximal function

$$
N_{D} F(x):=\sup _{x \in Q \in D} m_{Q}(F), \quad x \in Q_{1} .
$$

If $x \in Q_{1}$ lies outside $\bigcup_{Q \in D} Q$, it will be understood that $N_{D} F(x)=0$.

Claim 2.5. For $\mu$-a.e. $x \in Q_{0} \cap \operatorname{supp}(\mu)$, we have

$$
\begin{gathered}
\left|f(x)-m_{\left(Q_{0}\right)^{*}}(f)\right| \leq C M^{\sharp} f(x)+F(x), \\
F(x) \leq C\left(M^{\sharp} f(x)+N_{D} F(x)\right) .
\end{gathered}
$$

Proof. In view of $\delta\left(\left(Q_{0}\right)^{*},\left(2 Q_{0}\right)^{*}\right) \leq C$ the first inequality $(2.5)$ is obvious. To prove (2.6), first of all, we notice that, for $\mu$-a.e. $x \in Q_{0} \cap$ $\operatorname{supp}(\mu)$, there exists a sequence $\left\{Q_{k}\right\}_{k=1}^{\infty}$ of doubling cubes centered at $x$ with $\ell\left(Q_{k}\right) \rightarrow 0$ as $k \rightarrow \infty$ and (see [9])

$$
\lim _{k \rightarrow \infty} m_{Q_{k}}(F)=F(x) \text {. }
$$

Fix $x \in Q_{0} \cap \operatorname{supp}(\mu)$ satisfying (2.7). If $\widetilde{\delta}\left(x, Q_{1}\right)=\infty$, then $\ell\left(Q_{x, m}\right) \rightarrow 0$ as $m \rightarrow \infty$. Hence $F(x) \leq N_{D} F(x)$. If $\widetilde{\delta}\left(x, Q_{1}\right) \in(m A,(m+1) A]$ for some $m \in \mathbb{N}$, we have $\delta\left(Q, Q_{x, m}\right) \leq C$ for some sufficiently small doubling cube $Q$ centered at $x$ and contained in $Q_{x, m}$. Thus, we see that

$$
\begin{aligned}
& m_{Q}(F)=m_{Q}\left(\left|f-m_{\left(2 Q_{0}\right)^{*}}(f)\right|\right) \\
& \leq m_{Q}\left(\left|f-m_{Q}(f)\right|\right)+\left|m_{Q}(f)-m_{Q_{x, m}}(f)\right|+\left|m_{Q_{x, m}}(f)-m_{\left(2 Q_{0}\right)^{*}}(f)\right| \\
& \leq C\left(m_{Q}\left(\left|f-m_{Q}(f)\right|\right)+\frac{\left|m_{Q}(f)-m_{Q_{x, m}}(f)\right|}{1+\delta\left(Q, Q_{x, m}\right)}\right)+m_{Q_{x, m}}\left(\left|f-m_{\left(2 Q_{0}\right)^{*}}(f)\right|\right) \\
& \leq C\left(M^{\sharp} f(x)+N_{D} F(x)\right) .
\end{aligned}
$$

If $\widetilde{\delta}\left(x, Q_{1}\right) \leq A$, we have $\widetilde{\delta}\left(Q,\left(2 Q_{0}\right)^{*}\right) \leq C$, and hence there exists a sufficiently small doubling cube $Q$ centered at $x$ and contained in $Q_{1}$ such 
that

$$
m_{Q}(F) \leq m_{Q}\left(\left|f-m_{Q}(f)\right|\right)+\left|m_{Q}(f)-m_{\left(2 Q_{0}\right)^{*}}(f)\right| \leq C M^{\sharp} f(x) .
$$

These observations and (2.7) yield the claim.

By keeping Claim 2.5 in mind, it is now clear that the theorem follows from Claim 2.6 below.

Claim 2.6. We have

$$
\left(\int_{Q_{1}} N_{D} F(x)^{q} W(x)^{\alpha} d \mu(x)\right)^{1 / q} \leq C\left(\int_{Q_{1}} M^{\sharp} f(x)^{q} W(x)^{\alpha} d \mu(x)\right)^{1 / q} .
$$

We shall prove Claim 2.6 by means of the so-called good- $\lambda$ inequality.

LEMMA 2.7. There exists a constant $C$ such that

$$
\begin{aligned}
W^{\alpha}\left\{x \in Q_{1}: N_{D} F(x)>2 \lambda, M^{\sharp}\right. & f(x) \leq \eta \lambda\} \\
& \leq C \eta^{1-\alpha} W^{\alpha}\left\{x \in Q_{1}: N_{D} F(x)>\lambda\right\}
\end{aligned}
$$

for all $\lambda, \eta>0$.

Proof. Clearly, we may assume that $\eta>0$ is sufficiently small. Fixing $\eta>0$ sufficiently small, we set

$$
\begin{aligned}
& E_{\lambda}:=\left\{x \in Q_{1}: N_{D} F(x)>2 \lambda, M^{\sharp} f(x) \leq \eta \lambda\right\}, \\
& \Omega_{\lambda}:=\left\{x \in Q_{1}: N_{D} F(x)>\lambda\right\} .
\end{aligned}
$$

For all $x \in E_{\lambda}$, we can select a doubling cube $Q_{x}=Q_{z(x), m(x)} \in D, Q_{x} \ni x$, that satisfies $m_{Q_{x}}(F)>\frac{3}{2} \lambda$. If $m(x)=1$, we have $\delta\left(Q_{x},\left(2 Q_{0}\right)^{*}\right)<C$ and hence,

$$
m_{Q_{x}}(F) \leq m_{Q_{x}}\left(\left|f-m_{Q_{x}}(f)\right|\right)+\left|m_{Q_{x}}(f)-m_{\left(2 Q_{0}\right)^{*}}(f)\right| \leq C M^{\sharp} f(x) \leq C \eta \lambda .
$$

As a result we obtain $C \eta \lambda>\frac{3}{2} \lambda$, which is not possible for sufficiently small $\eta$. Replacing $Q_{z, m}$ by a younger one if necessary, we may assume that $m_{Q_{z, m}}(F)<\frac{3}{2} \lambda$ for any cube $Q_{z, m} \ni x$ with $m<m(x)$.

Let $S_{x}=Q_{z(x), m(x)-1}$. We claim that $m_{S_{x}}(F)>\lambda$ if $\eta$ is small enough. Indeed, noticing $\delta\left(Q_{x}, S_{x}\right) \leq 2 A$, we see that

$$
\begin{aligned}
m_{Q_{x}}(F) \leq & m_{Q_{x}}\left(\left|f-m_{Q_{x}}(f)\right|\right)+\left|m_{Q_{x}}(f)-m_{S_{x}}(f)\right| \\
& +\left|m_{S_{x}}(f)-m_{\left(2 Q_{0}\right)^{*}}(f)\right| \\
\leq & C M^{\sharp} f(x)+m_{S_{x}}(F) \\
\leq & C \eta \lambda+m_{S_{x}}(F) .
\end{aligned}
$$

This yields $m_{S_{x}}(F) \geq \frac{3}{2} \lambda-C \eta \lambda>\lambda$. Thus, we have

$$
3 \lambda / 2>m_{S_{x}}(F)>\lambda
$$

for sufficiently small $\eta$. 
Notice that $Q_{x} \subset \frac{1}{100} S_{x}$ by Lemma 2.3(i). By Besicovitch's covering lemma there exists a countable subset $\left\{x_{j}\right\}_{j \in J} \subset E_{\lambda}$ such that

$$
E_{\lambda} \subset \bigcup_{j \in J} S_{x_{j}} \text { and } \sum_{j \in J} \chi_{S_{x_{j}}} \leq C \chi_{\Omega_{\lambda}} .
$$

To simplify notation we write $S_{j}=S_{x_{j}}$ and $Q_{j}=Q_{x_{j}}$. Now we claim the following:

Claim 2.8. If $\eta$ is small enough, then

$$
W^{\alpha}\left(S_{j} \cap E_{\lambda}\right) \leq C \eta^{1-\alpha} W^{\alpha}\left(S_{j}\right) \quad \text { for all } j \in J \text {. }
$$

Let us temporarily accept Claim 2.8. Then (2.9) and the claim lead to

$$
W^{\alpha}\left(E_{\lambda}\right) \leq \sum_{j \in J} W^{\alpha}\left(S_{j} \cap E_{\lambda}\right) \leq C \eta^{1-\alpha} \sum_{j \in J} W^{\alpha}\left(S_{j}\right) \leq C \eta^{1-\alpha} W^{\alpha}\left(\Omega_{\lambda}\right),
$$

which proves Lemma 2.7 .

We are left with the task of proving Claim 2.8.

Proof of Claim 2.8. By Lemma 2.4 it suffices to show that

$$
\mu\left(S_{j} \cap E_{\lambda}\right) \leq C \eta \mu\left(S_{j}\right) .
$$

Let $y \in S_{j} \cap E_{\lambda}$. There exists a doubling cube $R_{y}=Q_{z(y), m(y)} \in D, R_{y} \ni y$, that satisfies $m_{R_{y}}(F)>2 \lambda$. We show that $\ell\left(R_{y}\right) \leq \frac{1}{8} \ell\left(S_{j}\right)$ whenever $\eta$ is sufficiently small. From Lemma 2.3(ii) we may assume that $m(y)<m\left(x_{j}\right)$. By Lemma 2.3(i) if $\ell\left(R_{y}\right)>\frac{1}{8} \ell\left(S_{j}\right)$, then $Q_{z(y), m(y)-1} \supset S_{j} \supset Q_{j}$. This and the fact that $m(y)-1<m\left(x_{j}\right)$ imply $3 \lambda / 2>m_{Q_{z(y), m(y)-1}}(F)$. Notice that $m_{R_{y}}(F)$ can be majorized by

$$
\begin{aligned}
m_{R_{y}}\left(\left|f-m_{R_{y}}(f)\right|\right)+\mid m_{R_{y}}(f)- & m_{Q_{z(y), m(y)-1}}(f) \mid \\
& +m_{Q_{z(y), m(y)-1}}\left(\left|f-m_{\left(2 Q_{0}\right)^{*}}(f)\right|\right),
\end{aligned}
$$

and consequently it can be bounded by $C M^{\sharp} f(y)+m_{Q_{z(y), m(y)-1}}(F)$. Thus,

$$
3 \lambda / 2>m_{Q_{z(y), m(y)-1}}(F) \geq m_{R_{y}}(F)-C M^{\sharp} f(y) \geq 2 \lambda-C \eta \lambda .
$$

Hence, if $\eta<1 / 3 C$, we must have $\ell\left(R_{y}\right) \leq \frac{1}{8} \ell\left(S_{j}\right)$. Thus,

$$
N_{D}\left(\chi_{\frac{5}{4} S_{j}} F\right)(y)>2 \lambda \quad \text { for all } y \in S_{j} \cap E_{\lambda} .
$$

From (2.8) we obtain $\left|m_{S_{j}}(f)-m_{\left(2 Q_{0}\right)^{*}}(f)\right| \leq 3 \lambda / 2$, and

$$
N_{D}\left(\chi_{\frac{5}{4} S_{j}}\left(f-m_{S_{j}}(f)\right)\right)(y)>\lambda / 2 \quad \text { for all } y \in S_{j} \cap E_{\lambda} .
$$

It follows from the weak- $(1,1)$ boundedness of $N_{D}$ that

$$
\begin{aligned}
\mu\left(S_{j} \cap E_{\lambda}\right) & \leq \mu\left\{y \in \mathbb{R}^{d}: N_{D}\left(\chi_{\frac{5}{4} S_{j}}\left(f-m_{S_{j}}(f)\right)\right)(y)>\lambda / 2\right\} \\
& \leq \frac{C}{\lambda} \int_{\frac{5}{4} S_{j}}\left|f(x)-m_{S_{j}}(f)\right| d \mu(x) .
\end{aligned}
$$


Recall that $S_{j}$ is doubling. Noticing that

$$
\begin{aligned}
& \int_{\frac{5}{4} S_{j}}\left|f(x)-m_{S_{j}}(f)\right| d \mu(x) \\
& \leq \int_{\frac{5}{4} S_{j}}\left|f(x)-m_{\left(\frac{5}{4} S_{j}\right)^{*}}(f)\right| d \mu(x)+\mu\left(S_{j}\right)\left|m_{\left(\frac{5}{4} S_{j}\right)^{*}}(f)-m_{S_{j}}(f)\right| \leq C \eta \lambda \mu\left(S_{j}\right),
\end{aligned}
$$

we conclude that $\mu\left(S_{j} \cap E_{\lambda}\right) \leq C \eta \mu\left(S_{j}\right)$.

We return to the proof of Claim 2.6.

Proof of Claim 2.6. Lemma 2.7 implies that

$$
\begin{aligned}
W^{\alpha}\{x & \left.\in Q_{1}: N_{D} F(x)>2 \lambda\right\} \\
& \leq C \eta^{1-\alpha} W^{\alpha}\left\{x \in Q_{1}: N_{D} f(x)>\lambda\right\}+W^{\alpha}\left\{x \in Q_{1}: M^{\sharp} f(x)>\eta \lambda\right\} .
\end{aligned}
$$

Multiplying both sides by $q \lambda^{q-1}$ and integrating against $\lambda$, we have, after a change of variables,

$$
\begin{aligned}
\frac{1}{2}\left(\int_{0}^{L} q \lambda^{q-1} W^{\alpha}\{x \in\right. & \left.\left.Q_{1}: N_{D} F(x)>\lambda\right\} d \lambda\right)^{1 / q} \\
\leq & \left(C \eta^{1-\alpha} \int_{0}^{L} q \lambda^{q-1} W^{\alpha}\left\{x \in Q_{1}: N_{D} F(x)>\lambda\right\} d \lambda\right)^{1 / q} \\
& +\frac{1}{\eta}\left(\int_{Q_{1}} M^{\sharp} f(x)^{q} W^{\alpha}(x) d \mu(x)\right)^{1 / q} .
\end{aligned}
$$

When $\eta$ is sufficiently small, we can bring the first term of the right-hand side to the left-hand side. As a consequence it follows that

$$
\begin{aligned}
\left(\int _ { 0 } ^ { L } q \lambda ^ { q - 1 } W ^ { \alpha } \left\{x \in Q_{1}: N_{D} F(x)>\right.\right. & \lambda\} d \lambda)^{1 / q} \\
& \leq C\left(\int_{Q_{1}} M^{\sharp} f(x)^{q} W(x)^{\alpha} d \mu(x)\right)^{1 / q} .
\end{aligned}
$$

Letting $L \rightarrow \infty$, we obtain the claim.

3. Application to vector-valued inequalities. Applying Theorem 1.2 , we can obtain some vector-valued inequalities. To formulate them we adopt the following notation. For a sequence $\left\{f_{j}\right\}_{j=1}^{\infty}$ of $\mu$-measurable functions and $q, r \geq 1$, we write

$$
\left\|f_{j}(x): l^{r}\right\|:=\left(\sum_{j=1}^{\infty}\left|f_{j}(x)\right|^{r}\right)^{1 / r}
$$


and

$$
\left\|f_{j}: L^{q}\left(l^{r}, \mu\right)\right\|:=\left(\int_{\mathbb{R}^{d}}\left\|f_{j}(x): l^{r}\right\|^{q} d \mu(x)\right)^{1 / q} .
$$

First of all, we need the following result (see [5, Theorem 1.7]).

LEMma 3.1. If $q, r \in(1, \infty)$, then

$$
\left\|N f_{j}: L^{q}\left(l^{r}, \mu\right)\right\| \leq C_{q, r}\left\|f_{j}: L^{q}\left(l^{r}, \mu\right)\right\| .
$$

Proposition 3.2. Let $q, r \in(1, \infty)$ and let $\left\{f_{j}\right\}_{j=1}^{\infty} \subset L_{\text {loc }}^{1}(\mu)$. Then there is a constant $C$ independent of $\left\{f_{j}\right\}_{j=1}^{\infty}$ so that, for every $Q_{0} \in \mathcal{Q}(\mu)$, $\left(\int_{Q_{0}}\left\|f_{j}(x)-m_{\left(Q_{0}\right)^{*}}\left(f_{j}\right): l^{r}\right\|^{q} d \mu(x)\right)^{1 / q} \leq C\left(\int_{\frac{3}{2} Q_{0}}\left\|M^{\sharp} f_{j}(x): l^{r}\right\|^{q} d \mu(x)\right)^{1 / q}$.

Proof. We may assume that $f_{j} \equiv 0$ for sufficiently large $j$ by the monotone convergence theorem. Take $1<s<\min (q, r)$ and let $t:=q / s, u:=r / s$. Take $\alpha$ slightly smaller than 1 so that $1<1 / \alpha<\min \left(t^{\prime}, u^{\prime}\right)$. We shall estimate

$$
\begin{aligned}
\mathrm{I} & :=\left(\int_{Q_{0}}\left\|f_{j}(x)-m_{\left(Q_{0}\right)^{*}}\left(f_{j}\right): l^{r}\right\|^{q} d \mu(x)\right)^{s / q} \\
& =\left(\int_{Q_{0}}\left\|\left|f_{j}(x)-m_{\left(Q_{0}\right)^{*}}\left(f_{j}\right)\right|^{s}: l^{u}\right\|^{t} d \mu(x)\right)^{1 / t}
\end{aligned}
$$

by a duality argument.

Take a vector-valued weight $\left(w_{1}, w_{2}, \ldots\right)$ supported on $Q_{0}$ and satisfying

$$
\begin{aligned}
& \mathrm{I}=\int_{Q_{0}}\left\|\left|f_{j}(x)-m_{\left(Q_{0}\right)^{*}}\left(f_{j}\right)\right|^{s} w_{j}(x)^{\alpha}: l^{1}\right\| d \mu(x), \\
& \left\|w_{j}^{\alpha}: L^{t^{\prime}}\left(l^{u^{\prime}}, \mu\right)\right\|=1 .
\end{aligned}
$$

Then it follows from Theorem 1.2 and Hölder's inequality that

$$
\begin{aligned}
& \mathrm{I} \leq \int_{\frac{3}{2} Q_{0}}\left\|M^{\sharp} f_{j}(x)^{s} W_{j}(x)^{\alpha}: l^{1}\right\| d \mu(x) \\
& \leq\left(\int_{\frac{3}{2} Q_{0}}\left\|M^{\sharp} f_{j}(x)^{s}: l^{u}\right\|^{t} d \mu(x)\right)^{1 / t} \cdot\left(\int_{\frac{3}{2} Q_{0}}\left\|W_{j}(x)^{\alpha}: l^{u^{\prime}}\right\|^{t^{\prime}} d \mu(x)\right)^{1 / t^{\prime}},
\end{aligned}
$$

where we have set

$$
W_{j}(x):=\sum_{k=1}^{\infty}(2 \beta)^{1-k} N^{k} w_{j}(x)
$$

and used the fact that $w_{j}(x) \leq W_{j}(x)$. Take for $\beta$ the constant $C_{\alpha t^{\prime}, \alpha u^{\prime}}$ in 
Lemma 3.1. Then Lemma 3.1, the definition of $W_{j}$ and (3.2) yield

$$
\begin{aligned}
\left(\int_{\frac{3}{2} Q_{0}}\left\|W_{j}(x)^{\alpha}: l^{u^{\prime}}\right\|^{t^{\prime}} d \mu(x)\right)^{1 / \alpha t^{\prime}} & \\
& =\left(\int_{\frac{3}{2} Q_{0}}\left\|W_{j}(x): l^{\alpha u^{\prime}}\right\|^{\alpha t^{\prime}} d \mu(x)\right)^{1 / \alpha t^{\prime}} \leq C .
\end{aligned}
$$

Piecing (3.1), (3.3) and (3.4) together, we obtain the desired conclusion.

The following corollary is a vector-valued extension of Theorem 1.1(ii).

Corollary 3.3. Let $f_{j} \in \mathrm{RBMO}$. For any cube $Q_{0} \in \mathcal{Q}(\mu)$ and $q, r \in$ $(1, \infty)$, there exists a constant $C$ independent of $f_{j}$ such that

$$
\left(\frac{1}{\mu\left(\frac{3}{2} Q_{0}\right)} \int_{Q_{0}}\left\|f_{j}(x)-m_{\left(Q_{0}\right)^{*}}\left(f_{j}\right): l^{r}\right\|^{q} d \mu(x)\right)^{1 / q} \leq C \sup _{x \in \mathbb{R}^{d}}\left\|M^{\sharp} f_{j}(x): l^{r}\right\| .
$$

We apply Proposition 3.2 to obtain a sharp maximal inequality on Morrey spaces.

Let $k>1,1 \leq q \leq p<\infty$ and $f \in L_{\text {loc }}^{q}(\mu)$. Then we say that $f$ belongs to the Morrey space $\mathcal{M}_{q}^{p}(k, \mu)$ provided

$$
\left\|f: \mathcal{M}_{q}^{p}(k, \mu)\right\|:=\sup _{Q \in \mathcal{Q}(\mu)} \mu(k Q)^{1 / p-1 / q}\left(\int_{Q}|f(x)|^{q} d \mu(x)\right)^{1 / q}<\infty .
$$

By applying Hölder's inequality to (3.5) it is easy to see that

$$
L^{p}(\mu)=\mathcal{M}_{p}^{p}(k, \mu) \subset \mathcal{M}_{q_{1}}^{p}(k, \mu) \subset \mathcal{M}_{q_{2}}^{p}(k, \mu)
$$

for $1 \leq q_{2} \leq q_{1} \leq p<\infty$. Let $k_{1}>k_{2}>1$. Then $\mathcal{M}_{q}^{p}\left(k_{1}, \mu\right)$ and $\mathcal{M}_{q}^{p}\left(k_{2}, \mu\right)$ coincide as sets and their norms are equivalent. More precisely (see [7, Proposition 1.1]),

$$
\left\|f: \mathcal{M}_{q}^{p}\left(k_{1}, \mu\right)\right\| \leq\left\|f: \mathcal{M}_{q}^{p}\left(k_{2}, \mu\right)\right\| \leq C_{d}\left(\frac{k_{1}-1}{k_{2}-1}\right)^{d}\left\|f: \mathcal{M}_{q}^{p}\left(k_{1}, \mu\right)\right\| .
$$

Nevertheless, for definiteness, we will assume $k=2$ in the definition and denote $\mathcal{M}_{q}^{p}(2, \mu)$ by $\mathcal{M}_{q}^{p}(\mu)$. For a sequence $\left\{f_{j}\right\}_{j=1}^{\infty}$ of $\mu$-measurable functions, we also write

$$
\left\|f_{j}: \mathcal{M}_{q}^{p}\left(l^{r}, \mu\right)\right\|:=\|\| f_{j}: l^{r}\left\|: \mathcal{M}_{q}^{p}(\mu)\right\| .
$$

The following proposition is a vector-valued extension of [8, Corollary 1.5].

Proposition 3.4. Let $\left\{f_{j}\right\}_{j=1}^{\infty} \subset L_{\text {loc }}^{1}(\mu)$. Suppose that $1<q \leq p<\infty$, $r \in(1, \infty)$ and there exists an increasing sequence of concentric doubling cubes $I_{1} \subset I_{2} \subset \cdots$ such that

$$
\lim _{k \rightarrow \infty} m_{I_{k}}\left(f_{j}\right)=0 \quad \text { for all } j \in \mathbb{N} \text { and } \bigcup_{k=1}^{\infty} I_{k}=\mathbb{R}^{d} \text {. }
$$


Then there exists a constant $C$ independent of $\left\{f_{j}\right\}_{j=1}^{\infty}$ such that

$$
\left\|f_{j}: \mathcal{M}_{q}^{p}\left(l^{r}, \mu\right)\right\| \leq C\left\|M^{\sharp} f_{j}: \mathcal{M}_{q}^{p}\left(l^{r}, \mu\right)\right\| .
$$

Proof. We may again assume that $f_{j} \equiv 0$ for sufficiently large $j$. Letting $R \in \mathcal{Q}(\mu)$, we shall estimate $\mu(2 R)^{1 / p-1 / q}\left(\int_{R}\left\|f_{j}(x): l^{r}\right\|^{q} d \mu(x)\right)^{1 / q}$. We begin by reducing the matter to the case when $R \in \mathcal{Q}(\mu, 2)$. It follows from Proposition 3.2 that

$$
\begin{aligned}
& \mu(2 R)^{1 / p-1 / q}\left(\int_{R}\left\|f_{j}: l^{r}\right\|^{q} d \mu\right)^{1 / q} \\
& \leq \mu(2 R)^{1 / p-1 / q}\left(\int_{R}\left\|f_{j}-m_{R^{*}}\left(f_{j}\right): l^{r}\right\|^{q} d \mu\right)^{1 / q}+\mu(R)^{1 / p}\left\|m_{R^{*}}\left(f_{j}\right): l^{r}\right\| \\
& \leq C \mu(2 R)^{1 / p-1 / q}\left(\int_{\frac{3}{2} R}\left\|M^{\sharp} f_{j}: l^{r}\right\|^{q} d \mu\right)^{1 / q}+\mu(R)^{1 / p}\left\|m_{R^{*}}\left(f_{j}\right): l^{r}\right\| \\
& \leq C\left\|M^{\sharp} f_{j}: \mathcal{M}_{q}^{p}\left(l^{r}, \mu\right)\right\|+\mu(R)^{1 / p}\left\|m_{R^{*}}\left(f_{j}\right): l^{r}\right\| .
\end{aligned}
$$

So we concentrate on estimating

$$
\mu(R)^{1 / p}\left\|m_{R^{*}}\left(f_{j}\right): l^{r}\right\| .
$$

We choose doubling cubes inductively. Let $R_{1}=R^{*}$ and $R_{m+1}=\left(2 R_{m}\right)^{*}$, $m \in \mathbb{N}$. Let $d$ be the distance between the center of $R_{1}$ and that of $I_{1}$. We select $m_{1} \in \mathbb{N}$ so large that $\ell\left(R_{m_{1}}\right) \geq 2 d$ and there exists some $I_{\kappa}, \kappa \in \mathbb{N}$, such that $R_{m_{1}} \subset I_{\kappa}, R_{m_{1}+1} \not \subset I_{\kappa}$ and

$$
\mu(R)^{1 / p}\left\|m_{I_{\kappa}}\left(f_{j}\right): l^{r}\right\| \leq\|\| M^{\sharp} f_{j}: l^{r}\left\|: \mathcal{M}_{q}^{p}(\mu)\right\| .
$$

Then a simple geometric observation shows that $R_{m_{1}} \subset I_{\kappa} \subset R_{m_{1}+3}$ and hence

$$
\delta\left(R_{m_{1}}, I_{\kappa}\right) \leq \delta\left(R_{m_{1}}, R_{m_{1}+3}\right) \leq C .
$$

For $i=1,2, \ldots$ we put

$$
M_{i}:=\left\{m \in \mathbb{N} \cap\left[1, m_{1}\right]: 2^{i-1} \mu(R) \leq \mu\left(R_{m}\right)<2^{i} \mu(R)\right\} .
$$

Deleting all empty sets from $\left\{M_{i}\right\}_{i=1,2, \ldots}$, we obtain $\left\{M_{i}\right\}_{i=i_{1}, \ldots, i_{\kappa^{\prime}}}$. Set

$$
a\left(i_{k}\right):=\min M_{i_{k}}, \quad b\left(i_{k}\right):=\max M_{i_{k}}, \quad k=1, \ldots, \kappa^{\prime} .
$$

Then we notice that $R_{b\left(i_{\kappa^{\prime}}\right)}=R_{m_{1}}$.

For $k=1, \ldots, \kappa^{\prime}-1$, from Lemma 2.1(iii) we see that

$$
\delta\left(R_{a\left(i_{k}\right)}, R_{b\left(i_{k}\right)}\right), \delta\left(R_{b\left(i_{k}\right)}, R_{a\left(i_{k+1}\right)}\right) \leq C,
$$


and hence $\delta\left(R_{a\left(i_{k}\right)}, R_{a\left(i_{k+1}\right)}\right) \leq C$. This implies that

$$
\begin{aligned}
& \mu(R)^{1 / p}\left\|m_{R_{a\left(i_{k}\right)}}\left(f_{j}\right)-m_{R_{a\left(i_{k+1}\right)}}\left(f_{j}\right): l^{r}\right\| \\
& \leq C 2^{-i_{k} / p} \mu\left(R_{a\left(i_{k}\right)}\right)^{1 / p-1 / q}\left(\int_{R_{a\left(i_{k}\right)}}\left\|M^{\sharp} f_{j}(x): l^{r}\right\|^{q} d \mu(x)\right)^{1 / q} \\
& \leq C 2^{-i_{k} / p}\left\|M^{\sharp} f_{j}: \mathcal{M}_{q}^{p}\left(l^{r}, \mu\right)\right\| .
\end{aligned}
$$

Similarly, from (3.9) we also have

$$
\mu(R)^{1 / p}\left\|m_{R_{a\left(i_{\kappa^{\prime}}\right)}}\left(f_{j}\right)-m_{I_{\kappa}}\left(f_{j}\right): l^{r}\right\| \leq C 2^{-i_{\kappa^{\prime}} / p}\left\|M^{\sharp} f_{j}: \mathcal{M}_{q}^{p}\left(l^{r}, \mu\right)\right\| .
$$

Applying the triangle inequality to (3.7), we finally have

$$
\begin{aligned}
\mu(R)^{1 / p} \| & m_{R^{*}}\left(f_{j}\right): l^{r} \| \\
\leq & \mu(R)^{1 / p} \sum_{k=1}^{\kappa^{\prime}-1}\left\|m_{R_{a\left(i_{k}\right)}}\left(f_{j}\right)-m_{R_{a\left(i_{k+1}\right)}}\left(f_{j}\right): l^{r}\right\| \\
& +\mu(R)^{1 / p}\left\{\left\|m_{R_{a\left(i_{\kappa^{\prime}}\right)}}\left(f_{j}\right)-m_{I_{\kappa}}\left(f_{j}\right): l^{r}\right\|+\left\|m_{I_{\kappa}}\left(f_{j}\right): l^{r}\right\|\right\} \\
\leq & C\left(\sum_{k=1}^{\kappa^{\prime}} 2^{-i_{k} / p}\right)\left\|M^{\sharp} f_{j}: \mathcal{M}_{q}^{p}\left(l^{r}, \mu\right)\right\|+\mu(R)^{1 / p}\left\|m_{I_{\kappa}}\left(f_{j}\right): l^{r}\right\| \\
\leq & C\left\|M^{\sharp} f_{j}: \mathcal{M}_{q}^{p}\left(l^{r}, \mu\right)\right\| .
\end{aligned}
$$

The proof is now complete.

4. Application to weighted inequalities. Applying Theorem 1.2, we can obtain a weighted sharp maximal inequality for Morrey spaces. For a weight $w$, we define the norm of the weighted Morrey space $\mathcal{M}_{q}^{p}(w, \mu)$ as

$$
\begin{array}{r}
\left\|f: \mathcal{M}_{q}^{p}(w, \mu)\right\|:=\sup _{Q \in \mathcal{Q}(\mu)} \mu(2 Q)^{1 / p-1 / q}\left(\int_{Q}|f(x)|^{q} w(x) d \mu(x)\right)^{1 / q}, \\
1 \leq q \leq p<\infty .
\end{array}
$$

The following proposition is a weighted version of [8, Corollary 1.5].

Proposition 4.1. Let $1 \leq q \leq p<\infty, 0<\alpha<q / p$ and $w, W$ be the same as Theorem 1.2. Suppose that $f \in L_{\mathrm{loc}}^{1}(\mu)$ and there exists an increasing sequence of concentric doubling cubes $I_{1} \subset I_{2} \subset \cdots$ such that

$$
\lim _{k \rightarrow \infty} m_{I_{k}}(f)=0 \quad \text { and } \quad \bigcup_{k=1}^{\infty} I_{k}=\mathbb{R}^{d} .
$$

Then there exists a constant $C$ independent of $f$ such that

$$
\left\|f: \mathcal{M}_{q}^{p}\left(W^{\alpha}, \mu\right)\right\| \leq C\left\|M^{\sharp} f: \mathcal{M}_{q}^{p}\left(W^{\alpha}, \mu\right)\right\| .
$$


Proof. The proof is similar to that of Proposition 3.4. We shall estimate

$$
\mu(2 R)^{1 / p-1 / q}\left(\int_{R}|f(x)|^{q} W(x)^{\alpha} d \mu(x)\right)^{1 / q}, \quad R \in \mathcal{Q}(\mu) .
$$

It follows from Theorem 1.2 that

$$
\begin{aligned}
\mu(2 R)^{1 / p-1 / q} & \left(\int_{R}|f(x)|^{q} W(x)^{\alpha} d \mu(x)\right)^{1 / q} \\
\leq & \mu(2 R)^{1 / p-1 / q}\left(\int_{R}\left|f(x)-m_{R^{*}}(f)\right|^{q} W(x)^{\alpha} d \mu(x)\right)^{1 / q} \\
& +\mu(R)^{1 / p-1 / q} W^{\alpha}(R)^{1 / q}\left|m_{R^{*}}(f)\right| \\
\leq & C \mu(2 R)^{1 / p-1 / q}\left(\int_{\frac{3}{2} R} M^{\sharp} f(x)^{q} W(x)^{\alpha} d \mu(x)\right)^{1 / q} \\
& +\mu(R)^{1 / p-1 / q} W^{\alpha}(R)^{1 / q}\left|m_{R^{*}}(f)\right| \\
\leq & C\left\|M^{\sharp} f: \mathcal{M}_{q}^{p}\left(W^{\alpha}, \mu\right)\right\|+\mu(R)^{1 / p-1 / q} W^{\alpha}(R)^{1 / q}\left|m_{R^{*}}(f)\right| .
\end{aligned}
$$

So the task is reduced to proving

$$
\mu(R)^{1 / p-1 / q} W^{\alpha}(R)^{1 / q}\left|m_{R^{*}}(f)\right| \leq C\left\|M^{\sharp} f: \mathcal{M}_{q}^{p}\left(W^{\alpha}, \mu\right)\right\| .
$$

Take $\kappa \in \mathbb{N}$ so large that

$$
\mu(R)^{1 / p-1 / q} W^{\alpha}(R)^{1 / q},\left|m_{I_{\kappa}}(f)\right| \leq\left\|M^{\sharp} f: \mathcal{M}_{q}^{p}\left(W^{\alpha}, \mu\right)\right\| .
$$

We will use the same definition and notation from the proof of Proposition 3.4. For $k=1, \ldots, \kappa^{\prime}-1$, we recall that $\delta\left(R_{a\left(i_{k}\right)}, R_{a\left(i_{k+1}\right)}\right) \leq C$, $\delta\left(R_{a\left(i_{\kappa^{\prime}}\right)}, I_{\kappa}\right) \leq C$ and, for $k=1, \ldots, \kappa^{\prime}$, we have

$$
\frac{W^{\alpha}(R)}{\mu(R)^{1-\alpha}} \leq(2 \beta)^{\alpha} \frac{W^{\alpha}\left(R_{a\left(i_{k}\right)}\right)}{\mu\left(R_{a\left(i_{k}\right)}\right)^{1-\alpha}}
$$

by Lemma 2.4. Combining these observations, we obtain

$$
\begin{aligned}
& \mu(R)^{1 / p-1 / q} W^{\alpha}(R)^{1 / q}\left|m_{R_{a\left(i_{k}\right)}}(f)-m_{R_{a\left(i_{k+1}\right)}}(f)\right| \\
&=\mu(R)^{1 / p-\alpha / q}\left(\frac{W^{\alpha}(R)}{\mu(R)^{1-\alpha}}\right)^{1 / q}\left|m_{R_{a\left(i_{k}\right)}}(f)-m_{R_{a\left(i_{k+1}\right)}}(f)\right| \\
& \leq C 2^{-(1 / p-\alpha / q) i_{k}} \mu\left(R_{a\left(i_{k}\right)}\right)^{1 / p-\alpha / q}\left(\frac{W^{\alpha}\left(R_{a\left(i_{k}\right)}\right)}{\mu\left(R_{a\left(i_{k}\right)}\right)^{1-\alpha}}\right)^{1 / q} \\
& \quad \times\left|m_{R_{a\left(i_{k}\right)}}(f)-m_{R_{a\left(i_{k+1}\right)}}(f)\right|
\end{aligned}
$$




$$
\begin{aligned}
\leq & C 2^{-(1 / p-\alpha / q) i_{k}} \mu\left(R_{a\left(i_{k}\right)}\right)^{1 / p}\left(\frac{W^{\alpha}\left(R_{a\left(i_{k}\right)}\right)}{\mu\left(R_{a\left(i_{k}\right)}\right)}\right)^{1 / q} \frac{1}{\mu\left(R_{a\left(i_{k}\right)}\right)} \\
& \times \int_{R_{a\left(i_{k}\right)}} M^{\sharp} f(x) d \mu(x) \\
\leq & C 2^{-(1 / p-\alpha / q) i_{k}} \mu\left(R_{a\left(i_{k}\right)}\right)^{1 / p-1 / q}\left(\int_{R_{a\left(i_{k}\right)}} M^{\sharp} f(x)^{q} W(x)^{\alpha} d \mu(x)\right)^{1 / q} \\
\leq & C 2^{-(1 / p-\alpha / q) i_{k}}\left\|M^{\sharp} f: \mathcal{M}_{q}^{p}\left(W^{\alpha}, \mu\right)\right\|,
\end{aligned}
$$

where we use $W^{\alpha}\left(R_{a\left(i_{k}\right)}\right) / \mu\left(R_{a\left(i_{k}\right)}\right) \leq\left(W\left(R_{a\left(i_{k}\right)}\right) / \mu\left(R_{a\left(i_{k}\right)}\right)\right)^{\alpha}$ and $(2.4)$. Similarly, we also have

$$
\begin{aligned}
\mu(R)^{1 / p-1 / q} W^{\alpha}(R)^{1 / q} \mid m_{R_{a\left({ }_{\kappa^{\prime}}\right)}} & (f)-m_{I_{\kappa}}(f) \mid \\
& \leq C 2^{-(1 / p-\alpha / q) \kappa^{\prime}}\left\|M^{\sharp} f: \mathcal{M}_{q}^{p}\left(W^{\alpha}, \mu\right)\right\| .
\end{aligned}
$$

Recall that $1 / p-\alpha / q>0$ by assumption. Applying the triangle inequality to $(4.2)$, we finally have

$$
\begin{aligned}
(4.2) \leq & \mu(R)^{1 / p-1 / q} W^{\alpha}(R)^{1 / q} \sum_{k=1}^{\kappa^{\prime}-1}\left|m_{R_{a\left(i_{k}\right)}}(f)-m_{R_{a\left(i_{k+1}\right)}}(f)\right| \\
& +\mu(R)^{1 / p-1 / q} W^{\alpha}(R)^{1 / q}\left\{\left|m_{R_{a\left(i_{\kappa^{\prime}}\right)}}(f)-m_{I_{\kappa}}(f)\right|+\left|m_{I_{\kappa}}(f)\right|\right\} \\
\leq & C\left(\sum_{k=1}^{\kappa^{\prime}} 2^{-(1 / p-\alpha / q) i_{k}}\right)\left\|M^{\sharp} f: \mathcal{M}_{q}^{p}\left(W^{\alpha}, \mu\right)\right\| \\
& +\mu(R)^{1 / p-1 / q} W^{\alpha}(R)^{1 / q}\left|m_{I_{\kappa}}(f)\right| \\
\leq & C\left\|M^{\sharp} f: \mathcal{M}_{q}^{p}\left(W^{\alpha}, \mu\right)\right\|,
\end{aligned}
$$

which is our desired estimate.

Acknowledgements. The first author is supported by Research Fellowships of the Japan Society for the Promotion of Science for Young Scientists. The second author is supported by the 21st century COE program at Graduate School of Mathematical Sciences, the University of Tokyo and the Fūjyukai foundation.

The authors are grateful to Professor S. Kuroda for having checked the manuscript carefully.

\section{References}

[1] D. Deng, Y. Han and D. Yang, Besov spaces with non-doubling measures, Trans. Amer. Math. Soc. 358 (2006), 2965-3001. 
[2] Y. Han and D. Yang, Triebel-Lizorkin spaces with non-doubling measures, Studia Math. 162 (2004), 105-140.

[3] F. Nazarov, S. Treil and A. Volberg, Cauchy integral and Calderón-Zygmund operators on nonhomogeneous spaces, Int. Math. Res. Not. 1997, no. 15, 703-726.

[4] F. Nazarov, S. Treil and A. Volberg, Weak type estimates and Cotlar inequalities for Calderón-Zygmund operators on nonhomogeneous spaces, Int. Math. Res. Not. 1998, no. 9, 463-487.

[5] Y. Sawano, Sharp estimates of the modified Hardy-Littlewood maximal operator on the nonhomogeneous space via covering lemmas, Hokkaido Math. J. 34 (2005), $435-458$.

[6] -, A vector-valued sharp maximal inequality on Morrey spaces with non-doubling measures, Georgian Math. J. 13 (2006), 153-172.

[7] Y. Sawano and H. Tanaka, Morrey spaces for non-doubling measures, Acta Math. Sin. (Engl. Ser.) 21 (2005), 1535-1544.

[8] - - - Sharp maximal inequalities and commutators on Morrey spaces with nondoubling measures, Taiwan. J. Math., to appear.

[9] X. Tolsa, BMO, $H^{1}$, and Calderón-Zygmund operators for non doubling measures, Math. Ann. 319 (2001), 89-149.

[10] -, Littlewood-Paley theory and the T(1) theorem with non-doubling measures, Adv. Math. 164 (2001), 57-116.

[11] - , The space $H^{1}$ for nondoubling measures in terms of a grand maximal operator, Trans. Amer. Math. Soc. 355 (2003), 315-348.

[12] - Painlevé's problem and the semiadditivity of analytic capacity, Acta Math. 190 (2003), 105-149.

[13] - Bilipschitz maps, analytic capacity, and the Cauchy integral, Ann. of Math. 355 (2003), 315-348.

Y. Sawano

Department of Mathematics

and Information Sciences

Tokyo Metropolitan University

Minami-Ohsawa 1-1, Hachioji-shi

Tokyo 192-0397, Japan

E-mail: yosihiro@tmu.ac.jp
H. Tanaka

Graduate School of Mathematical Sciences

The University of Tokyo 3-8-1 Komaba, Meguro-ku

Tokyo 153-8914, Japan

E-mail: htanaka@ms.u-tokyo.ac.jp

Received December 8, 2005

Revised version April 3, 2007 